neumoretroperitoneo de gran importancia en el diagnóstico de sospecha y que habitualmente es de muy difícil apreciación en la radiología simple (10). En nuestro paciente al agudizarse la clínica y aparecer el neumoperitoneo en las segundas radiografías realizadas, no fue necesario la realización de TAC puesto que esta situación era indicativa de precisar cirugía urgente.

El tratamiento adecuado en estos casos, consiste en solucionar de forma definitiva el problema que lo origina, generalmente mediante cirugía, asociado a un soporte vital adecuado y la administración de antibióticos de amplio espectro con adecuada cobertura para bacilos gramnegativos y anaerobios (10).

El pronostico de estos pacientes es generalmente malo por tratarse normalmente de enfermos de edad avanzada y patologías asociadas así como por el retraso diagnóstico con la consecuente evolución del cuadro clínico (2), aunque nuestro paciente evolucionó de forma favorable, probablemente por su edad joven y tratamiento relativamente precoz, dada la rápida transición del cuadro clínico con pocas horas de evolución.

M. J. Montoya Tabares, J. L. Martín, A. Ríos Zambudio', M. Carrasco Prats, J. López Espejo, G. Sánchez de la Villa

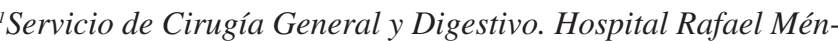
dez. Servicio de Cirugía General I. Hospital Universitario Virgen de la Arrixaca. Murcia

1. Bejvan SM, Gogwin JD. Pneumomediastinum: old signs and new signs. AJR Am J Roentgenol 1996 May; 166: 1041-8

2. Montero Fraile JA, López Altimiras J, Segura Movellan J, Puigdollers Pérez A, Ferez Bote F, Puey Bellosta J, Vilaseca Bellsola J. Perforación gastrointestinal asociada a gran enfisema subcutáneo. Rev Quir Esp 1988; 15: 153-5.

3. Evrard S, Mendoza L, Mutter D, Vetter D, Marescaux J. Massiive gas spread through a duodenal perforation after endoscopic sphincterotomy. Gastrointest Endosc 1993; 39: 817-8.

4. Chui PT, Gin T, Chung SC. Subcutaneous emphysema, pneumomediastinum and pneumothorax complicating laparoscopic vagotomy. Report of two cases. Anaesthesia 1993; 48: 978-81.

5. Briaud M, Barbier J, Morichau-Beauchant M, Blavon-Duchesne N, Matuchansky C. Pneumomédiastin, emphysème sous-cutané et ulcerè gastrique. Gastroenterol Clin Biol 1978; 2: 617-22.

6. Basak F, Kinaci E, Aksoy S, Aren A. Duodenal ulcer perforation and pneumotorax: a case report. Acta Chir Belg. 2006; 106: 344-5.

7. Utzig Mj, Foitzik T, Buhr HJ, Schneider P. Duodenal ulcer presenting as pneumomediastinum and pneumotorax. Case report. Zentralbl Chir. 2002; 127: 1091-3.

8. Von Heimburg RL, Alexander SJ, Sauer WG, Bernatz PE. Subcutaneous emphysema from perforated gastric ulcer. Ann Surg 1963; 158: 1-5.

9. O’Donoghue PD. Mediastinal surgical emphysema due to perforated duodenal ulcer. Lancet 1956; 270: 189.

10. Ríos Zambudio A, Piñero Madrona A, González Sanchez F, Parrilla Paricio P. Enfisema subcutáneo de miembro inferior como primera manifestación de una dehiscencia colónica. Rev Clin Esp 2002; 202: 571-2.

\section{Dolor e impotencia funcional de extremidad inferior izquierda y masa dolorosa en hemiabdomen izquierdo}

\section{Sr. Director:}

Hemos leído con especial atención la aportación de Cachorro San Pedro y cols. titulado Dolor e impotencia funcional de extremidad inferior derecha (1), donde sus autores presentan un caso de varón joven inmunocompetente con la clínica referida y con resultado de tuberculosis ósea intertrocantérea derecha. Nos gustaría presentar el caso de una mujer con dolor e impotencia funcional de extremidad inferior izquierda y masa en hemiabdomen izquierdo.

Se trata de una mujer de 27 años, natural de Ecuador, que ingresa tras cuadro de 3 meses de evolución de dolor e impotencia funcional progresiva en extremidad inferior izquierda, que en los últimos días advierte además una masa dolorosa en flanco izquierdo, precedido este cuadro por pérdida de $12 \mathrm{~kg}$ de peso y sensación distérmica vespertina en los últimos 8 meses. La exploración física nos reveló tumoración dolorosa de superficie lisa de 12 x $5 \mathrm{~cm}$ en cuadrante abdominal medio e inferior izquierdo y dolor a la flexo extensión de la cadera izquierda, así como en rotación externa. Analíticamente destaca elevación discreta de los reactantes de fase aguda (VSG de $26 \mathrm{~mm}$ en la $1^{\text {a }}$ hora, proteína $\mathrm{C}$ reactiva de $2.7 \mathrm{mg} / \mathrm{dL}$ ), perfil hepático y resto de parámetros analíticos, incluidos marcadores tumorales (a destacar $\mathrm{Ca}$ 19.9, CA 125) normales. El Mantoux resultó positivo (18 mm.) en la lectura a las 72 horas. El Ziehl en orina, esputos y jugo gástrico, al igual que serologías para equinoccocus y $\mathrm{VIH}$, resultaron también negativas. Realizada $\mathrm{Rx}$ tórax y abdomen, destaca patrón micronodular en ambos campos pulmonares superiores así como base pulmonar izquierda y abombamiento de la línea del músculo psoas izquierdo con esqueleto regional indemne, respectivamente. Ante los hallazgos se realiza tomografía axial computerizada (TAC) toraco abdominal evidenciando absceso en músculo psoas izquierdo de 15 × 6,5 x 6,2 ( $\mathrm{L}$ x T x AP) $\mathrm{cm}$ y numerosas adenopatías retroperitoneales de baja atenuación de localización peripancreática, interaortocava y paraaórticas izquierdas a la altura del hilio renal sin signos de espondilodiscistis aparente. Se procedió al drenaje percutáneo bajo control ecográfico, drenando $400 \mathrm{cc}$. de material purulento, y resultando a posteriori, cultivo en medio de Lowenstein positivo para Micobacterium tuberculosis complex. Se mantuvo drenaje durante 9 días, con desinserción casual sin recolocación por buena evolución en control ecográfico; se empleó régimen de isoniacida, rifampicina, piracinamida y etambutol 9 meses. Ante persistencia, pese a tratamiento antituberculostático con óptimo cumplimiento, fue necesario drenaje quirúrgico abierto 8 meses después por reducción incompleta del mismo.

El absceso de psoas es una entidad descrita por Mynter como psoitis a fines del siglo XIX. Se trata de un proceso poco frecuente cuya incidencia está aumentando en los últimos años, relacionado sin duda, con la enorme utilidad que las técnicas radiológicas (TAC y resonancia magnética) nos ofrecen en su diagnóstico; constituyen la segunda localización más frecuente de los abscesos musculares tras el absceso glúteo (2). Clásicamente han sido clasificados (3) como primarios si no se identifica foco primario desde el cual se ha producido la diseminación y su afectación se produce vía hematógena y secundarios a partir de la afectación infecciosa de órganos adyacentes, a destacar origen gastrointestinal, genitourinario y óseo (éste de especial importancia en el caso de los abscesos de psoas). Su presentación suele ser de curso insidioso y prolongado (4), especialmente en los de etiología tuberculosa y el dolor en flanco o hemiabdomen (91\%) (5), como en nuestro caso, seguido por dolor en cadera e ingle $(69,5 \%)$ son su forma de presentación preponderante, siendo la fiebre un signo inconstante y los reactantes de fase aguda, especialmente VSG y proteína $\mathrm{C}$ reactiva, marcadores evolutivos. En lo que a etiología se refiere destacan S. aureus y Mycobacterium tuberculosis como principales causas, sin olvidar la presencia en ocasiones de bacilos Gram negativos (E. coli) $(5,6)$. En el caso de la manifestación de esta rara forma de presentación extrapulmonar de la tuberculosis, ha variado en nuestro país con la irrupción de número de casos de VIH $(7,8)$ y con las nuevas formas de presentación de la tuberculosis en la población inmigrante (9) en las que la reactivación endógena y/o la infección exógena juegan un papel impor- 
tante. Las técnicas de imagen han dado un papel preponderante a la tomografía computerizada (10), si bien pueden existir signos indirectos como borramiento de las líneas del psoas en la Rx simple de abdomen que no han desdibujado su importancia. El tratamiento, además del antibiótico específico, en nuestra paciente antituberculoso, se debate entre las técnicas de drenaje percutáneo y el quirúrgico abierto, posiblemente inclinándose la balanza a favor del primero $(6,10)$, ya que minimiza los riesgos derivados de la cirugía mayor, pero sin poder obviar la sombra de la recidiva o el drenaje incompleto y la mala difusión de los antibióticos, que obliga, en ocasiones, a transformar las técnicas de drenaje percutáneo en drenaje quirúrgico abierto.

El absceso de psoas de etiología tuberculosa presenta por tanto, una clínica insidiosa, en muchas ocasiones precedida de síntomas generales comunes a la mayor parte de enfermedades sistémicas que demora su diagnóstico. Una correcta anámnesis y ante todo la sospecha clínica inicial, acortará el procedimiento diagnóstico y minimizará la morbilidad que de ello se deriva.

L. Mao Martín, M. A. Urbán Poza, R. Torres Gárate, E. Álvarez Rodríguez, B. Valle Borrego, F. Baquedano Sánchez, M. Díaz Ortiz

\section{Servicio de Medicina Interna I. Hospital Clínico San Carlos. Madrid}

1. Cachorro I, Pinilla J, Labarga P. Dolor e impotencia funcional de extremidad inferior derecha. An Med Interna (Madrid) 2005; 22: 498-499.

2. Martín M, García C, Gutiérrez M, Fernández M, González J, Hernández JL. Pyomyositis: retrospective review in a third-level hospital in the north of Spain. Enferm Infecc Microbiol Clin 2006; 24: 173-177.

3. Navarro V, Meseguer, V. Absceso del músculo psoas: ¿primario o secundario? Enferm Infecc Microbiol Clin 2002; 20: 235

4. Corti M, Palmieri O. J, Villafañe M. F, Muzzio E. Tuberculosis diseminada con absceso bilateral del psoas en un paciente con sida. Enferm Infecc Microbiol Clin 2004; 22: 197-200.

5. Penado S, Espina B, Campo J. F. Absceso de Psoas: descripción de una serie de 23 casos. Enferm Infecc Microbiol Clin 2001; 19: 257-260.

6. Navarro V, Meseguer A, Fernández F. Absceso del músculo psoas. Descripción de una serie de 19 casos. Enferm Infecc Microbiol Clin 1998; 16: 118-122.

7. Kozakis L, Ballachandran T. Bilateral psoas abscesses in an HIV-positive patient. Inter J STD \& AIDS 2004; 15: 841-843.

8. Navarro V, López F, González E, Gregori J, Muñoz A. Psoas abscess in patients infected with de human inmunodeficiency virus. Eur J Clin Microbiol Infect Dis 2004; 23: 661-3.

9. Sanz O, Caminero JA, Pérez JL. Tuberculosis e inmigración en España. Evidencias y controversias. Med Clin (Barc). 2006; 126: 259-269.

10. Pérez M, Rodríguez I, Lado FL, Rodríguez I. Absceso primario de Psoas. An Med Interna (Madrid) 2004; 21: 50-51.

\section{Desgaste profesional en atención continuada del área médica}

\section{Sr. Director:}

Desde su descripción el síndrome de fatiga o agotamiento por desgaste laboral, se consideró como específico de los profesionales que mantienen un contacto constante y directo con los beneficiarios de su trabajo, cuando entre ambos media una relación de ayuda o servicio. Es decir que las profesiones sanitarias son especialmente sensibles a esta patología incluida por la OMS en el capítulo XXI del CIE-10 como un factor de riesgo para la salud. El síndrome de desgaste, agotamiento profesional o burn out, tiene tres componentes esenciales en la situación de sobrecarga que implica: el agotamiento emocional, la despersonalización y la falta de realización profesional (1). Existe interés creciente en la literatura $(2,3)$ por esta patología que como se explica tiene trascendencia sobre la salud de los afectados y especialmente pude afectar también a los atendidos por los afectados con la trascendencia que eso tiene en los casos de sanitarios $(4,5)$. En los últimos años las condiciones laborales de los sanitarios han sufrido un empeoramiento creándose los contratos de atención continuada (guardias). Presentamos los datos sobre satisfacción laboral de estos profesionales en el Área de Medicina Interna en un hospital de tercer nivel del Servizo Galego de Saude (SERGAS). Se realizó un estudio descriptivo, transversal en 2006 siendo objeto de estudio los facultativos que vienen prestando servicio en asistencia continuada del Área Médica con contrato exclusivo en esta modalidad. Se autoadministró de forma anónima el cuestionario Maslach Burnout Inventory validado en su versión española de 22 items. Se analizaron también las características laborales de los objetos de estudio, la duración de la modalidad laboral, tiempo desde el final de la formación especializada, edad y sexo. Se incluyeron 5 casos, 3 mujeres y 2 varones de edades comprendidas entre los 32 y los 42 años. La media de años en asistencia continuada fue de 3,6 (rango 6-3 años). En la dimensión agotamiento emocional, el rango en las puntuaciones en el $40 \%$ de los entrevistados fue de 19-25 indicando un grado medio-elevado de burnout. En la dimensión despersonalización el 60\% presentó puntuaciones mayores de 10 indicado un grado elevado de desgaste. Finalmente en la realización personal el $80 \%$ presentó unas puntuaciones inferiores a 31 indicando un grado alto en esta dimensión.

En 1974 el psicólogo Freudemberguer introduce el término de burnout que posteriormente fue desarrollado difundiendo el concepto, sus características y evaluación por Maslach (6). El interés ha crecido en la medida en que ha aumentado la importancia de los servicios sociales y los dedicados al cuidado de seres humanos, los más afectados por esta patología. Los cambios en el mercado laboral son el principal desencadenante en el cambio radical en las condiciones laborales que también implica un aumento de este síndrome. La "flexibilización" (gran metáfora de precariedad) en el empleo y la intensificación en el trabajo han sido los principales mediadores en la transformación en la organización del trabajo. Las consecuencias son evidentes, en 2000 el estrés era el segundo trastorno de salud más frecuente entre los trabajadores de la Unión Europea (7), el 19\% de la población ocupada hacía turnos por la noche, el $47 \%$ trabajaba los sábados y el $24 \%$ los domingos. Los datos macroeconómicos y la presión de las pujantes nuevas economías que se caracterizan por una situación laboral más precaria, han supuesto la supresión de la jornada laboral de 35 horas en Francia (y probablemente a que nunca se plantee aquí). Se ha impuesto la denominada "directiva Bolkestein" de libre prestación de servicios sacralizando el dummping laboral en la UE. Además, todo indica que se van a intentar nuevas fórmulas de relación laboral ("proceso conjunto de extinción laboral", el nuevo despido; "contrato indefinido de duración limitada", etc. También en sanidad se sufren estos experimentos. Contrariamente a la opinión generalizada entre los no sanitarios que consideran a este medio laboral como privilegiado y cómodo, los experimentos contractuales están a la orden del día. Así se ofrecen: contratos por horas, por día, por "semana" (pero de lunes a viernes), por obra, por meses, etc exponentes del ingenio de los contratadores (por cierto muchos de ellos médicos, una verdadera "contratación creativa"). En nuestra comunidad autónoma además se ha creado en los últimos años lo que denominaremos "paro biológico" (paráfrasis de la actividad pesquera: se suspende el contrato prolongado el tiempo suficiente para que no se adquieran derechos que obliguen a convertirlo en indefinido o 